\title{
TRANSFORMATION FORMULA OF HIGHER ORDER INTEGRALS
}

\author{
TAO QIAN and TONGDE ZHONG
}

(Received 21 December 1998; revised 30 June 1999)

Communicated by P. G. Fenton

\begin{abstract}
By using integration by parts and Stokes' formula the authors give a new definition of the Hadamard principal value of higher order singular integrals on the complex hypersphere in $\mathbb{C}^{n}$. Then the transformation formula for the higher order singular integrals is deduced.
\end{abstract}

1991 Mathematics subject classification (Amer. Math. Soc.): primary 32A25, 31B10.

Keywords and phrases: complex hypersphere, higher order singular integral, transformation formula.

\section{Introduction}

The Plemelj formulas in relation to the Cauchy principal value singular integrals of one and several complex variables have very important applications to the theory of singular integral equations and the theory of elastic mechanics $([2,4,7])$. On the other hand, it arouses the question of how to define the principal value of higher order singular integrals when solving the Cauchy problem of hyperbolic partial differential equations and developing the theory of higher order singular integral equations. In fact, when one tries to find the limit value on the boundary of higher order derivatives of the Cauchy type integral of complex variables it will arouse the question as well. Firstly, in 1952 Hadamard defined, on the real axis, the Hadamard principal value using the idea of separating the finite part from the divergent integral ([3]). In 1957, Fox extended the idea to the case of higher order singular integrals in the complex plane $([1,5])$. The calculations of all those Hadamard principal values of higher order singular integrals, however, are too complicated and therefore very hard to be

Project supported by the Bilateral Science and Technology Collaboration Program of Australia 1998 and the Natural Science Foundation of China (No. 19771068).

(c) 2000 Australian Mathematical Society 0263-6115/2000\$A2.00+0.00 
extended to several complex variables. In [2], by using a crumble-some calculation a special case of higher order singular integrals on the complex hypersphere in $\mathbb{C}^{n}$ is discussed (see below formula (13) for $\beta=1 / 2, k=1$ ); and in [6] the general cases on the complex hypersphere in $\mathbb{C}^{n}$ is discussed. In [6] the method is simpler and some remarkable results are obtained. The definition of Hadamard principal value is still complicated and so it is hard to obtain more results using it. In this paper, using integration by parts and Stokes' formula, we inductively define higher order singular integrals through lower order singular integrals. In this way we uniformly define the Hadamard principal values of higher order singular integrals in one and several complex variables. The method agrees with the idea of Hadamard by separating the finite part from the divergent integral. Since the method is much simpler and straightforward, we can avoid tedious calculations and can effectively utilise the results of the standard Cauchy singular integrals. This enables us to obtain more results than in [2] and $[1,5,6]$.

For simplicity, we consider a higher order singular integral on the boundary of a bounded domain $D$ with smooth boundary in the complex plane

$$
\frac{1}{2 \pi i} \int_{\partial D} \frac{f(\zeta)}{(\zeta-\eta)^{2}} d \zeta, \quad \eta \in \partial D
$$

where $f(\zeta) \in H_{1}(\alpha), 0<\alpha \leq 1$, the set of functions whose first order derivatives $f^{\prime}(\zeta)$ belong to $H(\alpha)$, the class of functions of Hölder continuity with index $\alpha$. Singular integral (1) under the Cauchy principal value is divergent. Replacing $\eta \in \partial D$ by $z \in D$ in the above expression and using integration by parts, Stokes' formula then gives

$$
\begin{aligned}
\frac{1}{2 \pi i} \int_{\partial D} \frac{f(\zeta)}{(\zeta-z)^{2}} d \zeta & =\frac{1}{2 \pi i} \int_{\partial D}\left\{d\left[\left(\frac{-1}{\zeta-z}\right) f(\zeta)\right]-\left(\frac{-1}{\zeta-z}\right) d f(\zeta)\right\} \\
& =\frac{1}{2 \pi i} \int_{\partial D} \frac{f^{\prime}(\zeta)}{\zeta-z} d \zeta, \quad z \in D .
\end{aligned}
$$

Therefore the study of the Cauchy type integral $1 /(2 \pi i) \int_{\partial D} f(\zeta) /(\zeta-z)^{2} d \zeta$ is equivalent to the study of the Cauchy type integral $1 /(2 \pi i) \int_{\partial D} f^{\prime}(\zeta) /(\zeta-z) d \zeta$. Since $f^{\prime}(\zeta) \in H(\alpha)$, when $z=\eta \in \partial D$, the Cauchy principal value of the right hand side exists. If we denote the Hadamard principal value by FP and the Cauchy principal value by $P V$, respectively, then we may define

$$
\operatorname{FP} \frac{1}{2 \pi i} \int_{\partial D} \frac{f(\zeta)}{(\zeta-\eta)^{2}} d \zeta=\mathrm{PV} \frac{1}{2 \pi i} \int_{\partial D} \frac{f^{\prime}(\zeta)}{\zeta-\eta} d \zeta, \quad \eta \in \partial D .
$$

Let $f(\zeta) \in H_{n}(\alpha)$, where $H_{n}(\alpha)$ is the space of continuous functions whose $n$-th derivatives belongs to $H(\alpha)$. Then we may inductively define the Hadamard principal 
value of singular integrals of order $n$ to be

$$
\operatorname{FP} \frac{1}{2 \pi i} \int_{\partial D} \frac{f(\zeta)}{(\zeta-\eta)^{n+1}} d \zeta=\mathrm{PV} \frac{1}{2 \pi i n !} \int_{\partial D} \frac{f^{(n)}(\zeta)}{\zeta-\eta} d \zeta, \quad \eta \in \partial D
$$

This definition agrees with the original idea of Hadamard to separate the finite part from the divergent part. Moreover, in our definition the Hadamard principal value of higher order singular integrals are expressed by the Cauchy principal value of the standard Cauchy singular integral, so that we can conveniently utilise the results of the Cauchy principal value. As example, by denoting

$$
F(z)=\frac{1}{2 \pi i} \int_{\partial D} \frac{f(\zeta)}{(\zeta-z)^{n+1}} d \zeta=\frac{1}{2 \pi i n !} \int_{\partial D} \frac{f^{(n)}(\zeta)}{\zeta-z} d \zeta, \quad z \in D
$$

then Plemelj formula of the standard Cauchy singular integral implies

$$
\begin{aligned}
\lim _{z \rightarrow \eta^{ \pm}} F(z) & =\lim _{z \rightarrow \eta^{ \pm}} \frac{1}{2 \pi i n !} \int_{\partial D} \frac{f^{(n)}(\zeta)}{\zeta-z} d \zeta \\
& = \pm \frac{1}{2(n !)} f^{(n)}(\eta)+\frac{1}{2 \pi i n !} \mathrm{PV} \int_{\partial D} \frac{f^{(x)}(\zeta)}{\zeta-\eta} d \zeta \\
& = \pm \frac{1}{2(n !)} f^{(x)}(\eta)+\frac{1}{2 \pi i} \operatorname{FP} \int_{\partial D} \frac{f(\zeta)}{(\zeta-\eta)^{n+1}} d \zeta,
\end{aligned}
$$

where $z \rightarrow \eta^{ \pm}$denotes the limit procedure that $z$ tends to $\eta$ from inside or outside $D$, respectively.

This gives the Plemelj formula of higher order singular integrals (see [1])

$$
\begin{aligned}
& F^{+}(\eta)=\frac{1}{2(n !)} f^{(n)}(\eta)+\frac{1}{2 \pi i} \mathrm{FP} \int_{\partial D} \frac{f(\zeta)}{(\zeta-\eta)^{n+1}} d \zeta, \quad \eta \in \partial D \\
& F^{-}(\eta)=-\frac{1}{2(n !)} f^{(n)}(\eta)+\frac{1}{2 \pi i} \operatorname{FP} \int_{\partial D} \frac{f(\zeta)}{(\zeta-\eta)^{n+1}} d \zeta, \quad \eta \in \partial D
\end{aligned}
$$

where

$$
F(z)=\frac{1}{2 \pi i} \int_{\partial D} \frac{f(\zeta)}{(\zeta-z)^{n+1}} d \zeta, \quad z \in D
$$

If $\phi(\zeta, \xi)$ is defined on $\partial D \times \partial D$ and $\partial^{k+l+1} \phi(\zeta, \xi) / \partial \zeta^{p} \partial \xi^{k+l+1-p} \in H(\alpha), p=$ $0,1, \ldots, k+l+1$, then we can obtain transformation formula of higher order singular integrals ([5])

(7) $\int_{\partial D} \frac{d \zeta}{(\zeta-\eta)^{l+1}} \int_{\partial D} \frac{\phi(\zeta, \xi)}{(\xi-\zeta)^{k+1}} d \xi$ 


$$
=-\frac{\pi^{2}}{k ! l !} \sum_{p=0}^{l} C_{l}^{p} \phi^{(p, k+l-p)}(\eta, \eta)+\int_{\partial D} d \xi \int_{\partial D} \frac{\phi(\zeta, \xi)}{(\zeta-\eta)^{l+1}(\xi-\zeta)^{k+1}} d \zeta,
$$

where $C_{l}^{p}=l ! /(p !(l-p) !), 0 \leq p \leq l$.

The proof of the formula is based on the definition of Hadamard principle and the transformation formula of the standard Cauchy singular integral ([4]), and proceeds as follows

$$
\begin{aligned}
& \int_{\partial D} \frac{d \zeta}{(\zeta-\eta)^{l+1}} \int_{\partial D} \frac{\phi(\zeta, \xi)}{(\xi-\zeta)^{k+1}} d \xi \\
& =\frac{1}{k !} \int_{\partial D} \frac{d \zeta}{(\zeta-\eta)^{l+1}} \int_{\partial D} \frac{\phi^{(0, k)}(\zeta, \xi)}{\xi-\zeta} d \xi \\
& =\frac{1}{k ! l !} \int_{\partial D} \frac{d \zeta}{\zeta-\eta} \int_{\partial D} \frac{\partial^{l}}{\partial \zeta^{l}}\left[\frac{1}{\xi-\zeta} \phi^{(0 . k)}(\zeta, \xi)\right] d \xi \\
& =\frac{1}{k ! l !} \int_{\partial D} \frac{d \zeta}{\zeta-\eta} \int_{\partial D}\left[\sum_{p=0}^{l} C_{l}^{p} \frac{\partial^{p}}{\partial \zeta^{p}}\left(\frac{1}{\xi-\zeta}\right) \phi^{(l-p, k)}(\zeta, \xi)\right] d \xi \\
& =\frac{1}{k ! l !} \int_{\partial D} \frac{d \zeta}{\zeta-\eta} \int_{\partial D}\left[\sum_{p=0}^{l} C_{l}^{p} \frac{p !}{(\xi-\zeta)^{p+1}} \phi^{(l-p, k)}(\zeta, \xi)\right] d \xi \\
& =\frac{1}{k ! l !} \int_{\partial D} \frac{d \zeta}{\zeta-\eta} \int_{\partial D} \sum_{p=0}^{l} C_{l}^{p} \frac{\phi^{(l-p, k+p)}(\zeta, \xi)}{\xi-\zeta} d \xi \\
& =\frac{1}{k ! l !} \int_{\partial D} \frac{d \zeta}{\zeta-\eta} \int_{\partial D} \sum_{p=0}^{l} C_{l}^{p} \frac{\phi^{(p, k+l-p)}(\zeta, \xi)}{\xi-\zeta} d \xi \\
& =-\frac{\pi^{2}}{k ! l !} \sum_{p=0}^{l} C_{l}^{p} \phi^{(p, k+l-p)}(\eta, \eta)+\frac{1}{k ! l !} \int_{\partial D} d \xi \int_{\partial D} \frac{\sum_{p=0}^{l} C_{l}^{p} \phi^{(p . k+l-p)}(\zeta, \xi)}{(\zeta-\eta)(\xi-\zeta)} d \zeta \\
& =-\frac{\pi^{2}}{k ! l !} \sum_{p=0}^{l} C_{l}^{p} \phi^{(p, k+l-p)}(\eta, \eta)+\int_{\partial D} d \xi \int_{\partial D} \frac{\phi(\zeta, \xi)}{(\zeta-\eta)^{l+1}(\xi-\zeta)^{k+1}} d \zeta,
\end{aligned}
$$

where the last equality follows from integration by parts first with respect to $d \zeta$ and then with respect to $d \xi$.

Define the operator

$$
S_{n} \phi=\frac{1}{\pi i} \int_{\partial D} \frac{\phi(\zeta)}{(\zeta-\eta)^{n+1}} d \zeta, \quad \eta \in \partial D
$$

If we take $\phi(\zeta, \xi)=\phi(\xi)$ in (7), or alternatively by invoking the definition (3) and applying the composite formula of one complex variable ([4]) directly, we obtain the following composite formula of higher order singular integrals ([5])

$$
S_{m} S_{n} \phi(\eta)=\frac{1}{n ! m !} \phi^{(n+m)}(\eta), \quad \eta \in \partial D .
$$


By using the composite formula we can solve singular integral equations of higher orders. As example, we consider the solution of the equation

$$
a S_{m} \phi+b S_{n} \phi=\psi
$$

for $\phi(\zeta) \in H_{n+m}(\alpha)$, where $\psi$ is a given function in $H_{n}(\alpha)$ or in $H_{m}(\alpha)$, and $a, b$ are constants. According to the definition of the Hadamard principal value (3), the equation (10) is actually a differential integral equation. The solution may be obtained, for instance, by applying the operator $S_{m}$ to the both sides of (10) and then applying the composite formula (9). Then we have

$$
\frac{a}{(m !)^{2}} \phi^{(2 m)}+\frac{b}{n ! m !} \phi^{(n+m)}=S_{m} \psi \text {. }
$$

Thus we need to solve an ordinary differential equation. Under suitable boundary conditions, the solution function $\phi$ can be obtained uniquely. By following the same line of reasoning we can discuss more general forms of higher order singular integral equations with constant or variable coefficients.

In the following section we will use the same idea to study higher order singular integrals on the hypersphere in $\mathbb{C}^{n}$.

\section{Hadamard principal value of higher order singular integrals on the complex hypersphere}

Let

$$
B=\left\{z \in \mathbb{C}^{n} \mid z \bar{z}^{\prime}<1\right\}, \quad S=\left\{z \in \mathbb{C}^{n} \mid z \bar{z}^{\prime}=1\right\}
$$

be the unit ball and its boundary, the complex hypersphere in $\mathbb{C}^{n}$, where for any $z=\left(z_{1}, \ldots, z_{n}\right), \xi=\left(\xi_{1}, \ldots, \xi_{n}\right), z \bar{\xi}^{\prime}=z_{1} \bar{\xi}_{1}+\cdots+z_{n} \overline{\xi_{n}}$. The area element of the complex hypersphere $S$ is denoted by $d S(u)=\dot{u}$. The Cauchy formula for any analytic function in the unit ball $B$ continuous to its boundary is

$$
f(z)=\frac{1}{\omega_{n}} \int_{s} \frac{f(u)}{\left(1-z \vec{u}^{\prime}\right)^{n}} \dot{u},
$$

where

$$
\omega_{n}=\int_{S} d S(u)=\frac{2 \pi^{n}}{\Gamma(n)} .
$$

In order to study higher order singular integrals on the complex hypersphere $S$, we will first study the following form of higher order Cauchy type integral

$$
F(x)=\frac{1}{\omega_{n}} \int_{s} \frac{f(u)}{\left(1-z \bar{u}^{\prime}\right)^{n-\beta+k}} \dot{u},
$$

where $k$ is a positive integer, $0 \leq \beta<1$. We have the following lemma ([6]). 
LEMMA 1. Let $f \in H_{k}(\alpha)$ on $\bar{B}$ and $L$ be the operator defined by

$$
\begin{aligned}
(L f)(u) & =\frac{1}{\bar{u}_{1}}\left[u_{1} \frac{\partial f}{\partial u_{1}}(u)-\bar{u}_{1} \frac{\partial f}{\partial \bar{u}_{1}}(u)+f(u)\right], \quad u_{1} \neq 0, \\
L^{i} f & =L\left(L^{j-1} f\right), \quad L^{0}(f)=f .
\end{aligned}
$$

Then for any $z \in B, z_{1} \neq 0$, we have

$$
\begin{aligned}
\int_{S} \frac{f(u)}{\left(1-z \bar{u}^{\prime}\right)^{n-\beta+k}} \dot{u} \\
\quad=\frac{1}{(n-\beta+k-1)(n-\beta+k-2) \cdots(n-\beta) z_{1}^{k}} \int_{S} \frac{\left(L^{k} f\right)(u)}{\left(1-z \bar{u}^{\prime}\right)^{n-\beta}} \dot{u}
\end{aligned}
$$

The proof is a simplification of [6].

PROOF. For the complex hypersphere $S$ we have

$$
\bar{u}_{1} d u_{1}+\cdots+\bar{u}_{n} d u_{n}+u_{1} d \bar{u}_{1}+\cdots+u_{n} d \bar{u}_{n}=0 .
$$

In the subset of $S$ where $u_{1} \neq 0$, we have

$$
d \bar{u}_{1} \equiv-\frac{u_{j}}{u_{1}} d \bar{u} j \bmod \left(d u_{1}, \ldots, d u_{n}, d \bar{u}_{2}, \ldots,\left[d \bar{u}_{j}\right], \ldots, d \bar{u}_{n}\right), \quad j \geq 2,
$$

then the area element of $S$ can be expressed by

$$
\dot{u}=d S(u)=\frac{1}{2^{n-1} i^{n} u_{1}} d u_{1} \cdots d u_{n} d \bar{u}_{2} \cdots d \bar{u}_{n} .
$$

Therefore, when $u_{1} \neq 0$, for any positive integer $m$, by Stokes' formula we have

$$
\begin{aligned}
& \int_{S} \frac{f(u)}{\left(1-z \bar{u}^{\prime}\right)^{m+1}} \dot{u} \\
&=\frac{1}{2^{n-1} i^{n}} \int_{S} \frac{f(u)}{u_{1}\left(1-z \bar{u}^{\prime}\right)^{m+1}} d u_{1} \cdots d u_{n} d \bar{u}_{2} \cdots d \bar{u}_{n} \\
&=\frac{1}{2^{n-1} i^{n} m} \int_{S} \frac{f(u)}{u_{1}}\left(-\frac{u_{1}}{z_{1} \bar{u}_{1}}\right) d\left[\frac{1}{\left(1-z \bar{u}^{\prime}\right)^{m}}\right] d u_{2} \cdots d u_{n} d \bar{u}_{2} \cdots d \bar{u}_{n} \\
&= \frac{1}{2^{n-1} i^{n} m z_{1}} \int_{S} \frac{1}{\left(1-z \bar{u}^{\prime}\right)^{m}} d\left(\frac{f(u)}{\bar{u}_{1}}\right) d u_{2} \cdots d u_{n} d \bar{u}_{2} \cdots d \bar{u}_{n} \\
&-\frac{1}{2^{n-1} i^{n} m z_{1}} \int_{S} d\left[\frac{1}{\left(1-z \bar{u}^{\prime}\right)^{m}}\left(\frac{f(u)}{\bar{u}_{1}}\right)\right] d u_{2} \cdots d u_{n} d \bar{u}_{2} \cdots d \bar{u}_{n} \\
&= \frac{1}{m z_{1}} \int_{S} \frac{(L f)(u)}{\left(1-z \bar{u}^{\prime}\right)^{m}} \dot{u} .
\end{aligned}
$$

By induction (14) follows immediately. 
Equation (14) implies that the study of higher order Cauchy type integrals on the unit ball $B$ may be transferred to the study of the standard Cauchy integral on the unit ball $B$. Since when $z=\eta \in S$, the principal value of the singular integral on the right hand side of (14) exists ([2]), we may define the Hadamard principal value of higher order singular integrals on the complex hypersphere as follows.

DEFINITION 1. If $f$ is a function defined on the complex hypersphere whose all $k$ th partial derivatives are of Hölder continuity of degree $\alpha, 0<\alpha \leq 1$, then for $k \geq 1$ and $0 \leq \beta<1$, we define

$$
\begin{aligned}
\mathrm{FP} \frac{1}{\omega_{n}} \int_{S} \frac{f(u)}{\left(1-v \bar{u}^{\prime}\right)^{n-\beta+k}} \dot{u}= & \frac{1}{(n-\beta+k-1)(n-\beta+k-2) \cdots(n-\beta) v_{1}^{k} \omega_{n}} \\
& \times \mathrm{PV} \int_{S} \frac{\left(L^{k} f\right)(u)}{\left(1-v \bar{u}^{\prime}\right)^{n-\beta}} \dot{u}, \quad v \in S .
\end{aligned}
$$

The above definition of the Hadamard principal value is obviously simpler and clearer than that in [6]. This definition has the following advantages:

(1) It automatically discards the divergent part in the sense of Hadamard. It only keeps the finite part so that we can save the complicated calculations involved in the theory and the applications.

(2) It is defined on a general point $v$ on $S$.

(3) The Hadamard principal value is in terms of the Cauchy principal value. This gives us the convenience to utilise the results of the Cauchy principal value.

As example, using Plemelj formula of the standard Cauchy singular integral on $S$ ([2]), we have

THEOREM 1. Under the assumptions of Definition 1, if $z$ approaches $\eta \in S$ from the inner part of $S$ satisfying

$$
\frac{d(\eta, v)}{d(z, S)} \leq M<\infty
$$

where $M$ is a constant, then the following Plemelj formula holds

$$
\begin{aligned}
\lim _{z \rightarrow \eta^{+}} & \frac{1}{\omega_{n}} \int_{S} \frac{f(u)}{\left(1-z \bar{u}^{\prime}\right)^{n-\beta+k}} \dot{u} \\
= & \operatorname{FP} \frac{1}{\omega_{n}} \int_{S} \frac{f(u)}{\left(1-\eta \bar{u}^{\prime}\right)^{n-\beta+k}} \dot{u} \\
& +\frac{1}{2} \frac{1}{(n-\beta+k-1)(n-\beta+k-2) \cdots(n-\beta) \eta_{1}^{k} \omega_{n}}\left(L^{k} f\right)(\eta) .
\end{aligned}
$$


ProOF. Since

$$
\begin{aligned}
\lim _{z \rightarrow \eta^{+}} & \frac{1}{\omega_{n}} \int_{S} \frac{f(u)}{\left(1-z \vec{u}^{\prime}\right)^{n-\beta+k}} \dot{u} \\
= & \lim _{z \rightarrow \eta^{+}} \frac{1}{(n-\beta+k-1)(n-\beta+k-2) \cdots(n-\beta) z_{1}^{k} \omega_{n}} \int_{S} \frac{\left(L^{k} f\right)(u)}{\left(1-z \bar{u}^{\prime}\right)^{n-\beta}} \dot{u} \\
= & \frac{1}{(n-\beta+k-1)(n-\beta+k-2) \cdots(n-\beta) \eta_{1}^{k} \omega_{n}} \int_{S} \frac{\left(L^{k} f\right)(u)}{\left(1-\eta \bar{u}^{\prime}\right)^{n-\beta}} \dot{u} \\
& +\frac{1}{2} \frac{1}{(n-\beta+k-1)(n-\beta+k-2) \cdots(n-\beta) \eta_{1}^{k} \omega_{n}}\left(L^{k} f\right)(\eta),
\end{aligned}
$$

the identity (15) follows from Definition 1.

\section{Transformation formula}

THEOREM 2 (Higher order Poincaré-Bertrand formula). If $\phi(u, w) \in H_{k+1}(\alpha)$, $0<\alpha \leq 1$, then for $k \geq 1$ we have

$$
\begin{aligned}
& \left(\frac{2}{\omega_{n}}\right)^{2} \int_{S} \frac{1}{\left(1-v \bar{u}^{\prime}\right)^{n+1}} \dot{u} \int_{S} \frac{\phi(u, \omega)}{\left(1-u \bar{\omega}^{\prime}\right)^{n+k}} \dot{\omega} \\
& =\left[\chi_{k} \phi\right](v, v, v)+\left(\frac{2}{\omega_{n}}\right)^{2} \int_{S} \dot{\omega} \int_{S} \frac{\left[\chi_{k} \phi\right](v, u, \omega)}{\left(1-v \bar{u}^{\prime}\right)^{n}\left(1-u \bar{\omega}^{\prime}\right)^{n}} \dot{u},
\end{aligned}
$$

where

(17) $\left[\chi_{k} \phi\right](v, u, \omega)$

$$
\begin{aligned}
= & \frac{1}{(n+k-1)(n+k-2) \cdots n \cdot n v_{1} \bar{u}_{1} u_{1}^{k}} \\
& \times\left[\left(\omega_{1} \frac{\partial}{\partial \omega_{1}}-\bar{\omega}_{1} \frac{\partial}{\partial \omega_{1}}\right)+\left(u_{1} \frac{\partial}{\partial u_{1}}-\bar{u}_{1} \frac{\partial}{\partial \bar{u}_{1}}\right)+(1-k)\right] L_{\omega}^{k} \phi(u, \omega) .
\end{aligned}
$$

The higher order singular integral on the left-hand side of (16) takes the Hadamard principal value as in Definition 1 and the singular integral on the right hand side takes the Cauchy principal value.

Proof. By Definition 1 we have

$$
\begin{aligned}
\left(\frac{2}{\omega_{n}}\right)^{2} & \int_{S} \frac{1}{\left(1-v \bar{u}^{\prime}\right)^{n+1}} \dot{u} \int_{S} \frac{\phi(u, \omega)}{\left(1-u \bar{\omega}^{\prime}\right)^{n+k}} \dot{\omega} \\
& =\left(\frac{2}{\omega_{n}}\right)^{2} \int_{S} \frac{1}{\left(1-v \bar{u}^{\prime}\right)^{n}} \dot{u} \int_{S} \frac{1}{(n+k-1)(n+k-2) \cdots n \cdot n v_{1}}
\end{aligned}
$$




$$
\begin{aligned}
& \times L_{u}\left[\frac{L_{\omega}^{k} \phi(u, \omega)}{u_{1}^{k}\left(1-u \bar{\omega}^{\prime}\right)^{n}}\right] \dot{\omega} \\
= & \left(\frac{2}{\omega_{n}}\right)^{2} \int_{S} \frac{1}{\left(1-v \bar{u}^{\prime}\right)^{n}} \dot{u} \int_{S}\left[\chi_{k} \phi\right](v, u, \omega) \frac{1}{\left(1-u \bar{\omega}^{\prime}\right)^{n}} \dot{\omega} .
\end{aligned}
$$

Formula (17) follows then from the Poincaré-Bertrand formula for the standard Cauchy singular integral $[7,2.13 .5]$.

REMARK. (1) Similarly we can obtain the transformation formula with the order of singularity of the outer integral of (16) being greater than 1 , but the expression is complex.

(2) When $\phi(u, \omega)=\phi(\omega)$, to be consistent with the formula (17), we should have

$$
\begin{aligned}
{\left[\chi_{k} \phi\right](v, u, \omega)=} & \frac{1}{(n+k-1)(n+k-2) \cdots n \cdot n v_{1} \bar{u}_{1} u_{1}^{k}} \\
& \times\left[\left(\omega_{1} \frac{\partial}{\partial \omega_{1}}-\bar{\omega}_{1} \frac{\partial}{\partial \omega_{1}}\right)+(1-k)\right] L_{\omega}^{k} \phi(\omega)
\end{aligned}
$$

which still depends on three variables $v, u$ and $\omega$. This makes the argument for proving the composite formula in the one variable case fail to work. In fact, there is no composite formula for higher order singular integrals on the complex hypersphere in this setting.

\section{Acknowledgement}

The second author wishes to express his sincere gratitude for the hospitality and good working environment provided by the University of New England during his visit to Armidale.

\section{References}

[1] C. Fox, 'A generalization of the Cauchy principal value', Canad. J. Math. 9 (1957), 110-117.

[2] S. Gong, Integrals of Cauchy type on the ball (International Press, Cambridge MA, 1993).

[3] J. Hadamard, Lectures on Cauchy's problem in linear partial differential equations (Dover, New York, 1953).

[4] N. I. Muskhelishvili, Singular integral equations (Noordhoff, Groningen, 1953).

[5] C-Y. Wang, 'Hadamard's principal value of the singular integral', Chinese Ann. Math. 3 (2) (1982), 195-202.

[6] X-Q. Wang, Singular integrals and analyticity theorems in several complex variables (Ph. D. Thesis, Uppsala University, Sweden, 1990). 
[7] T-D. Zhong, Integral representation of functions of several complex variables and multidimensional singular integral equations (Xiamen University Press, Xiamen, 1986) (Chinese).

School of Mathematics and Computer Sciences

The University of New England

Armidale, NSW 2351

Australia

e-mail: tao@mcs.une.edu.au
Institute of Mathematics

Xiamen University

Xiamen 361005

P. R. China 